\title{
Percepção da qualidade da informação
}

Ronaldo Ronan Oleto

Doutor em ciência da informação pela UFMG.

E-mail: ronaldo.ronan@fjp.mg.gov.br

\section{Resumo}

A qualidade da informação tem atributos/conceitos que alguns autores usam para melhor compreendê-la. Outros são críticos quanto à proposta de se atribuir qualidade à informação. No texto, algumas linhas provocativas quanto à percepção desses atributos pelo usuário da informação são discutidas. Há, também, a listagem de alguns atributos com os seus respectivos entendimentos, separados pela perspectiva da abordagem: se centrado no produto ou centrado no usuário. Se centrada no produto, a informação é tida como um bem ou coisa; se centrada no usuário, ela é valorizada ante a necessidade dele. Em pesquisa com uma amostra de usuários da informação na atividade de administração de imóveis, o autor não encontrou, de forma clara, o entendimento dos conceitos sobre os atributos da qualidade da informação. Apresentados alguns conceitos/ atributos a esses usuários, observou-se que a percepção deles tangenciava o senso comum e era fortemente influenciada pela sinonímia.

\section{Palavras-chave}

Ciência da informação. Qualidade da informação. Percepção da qualidade da informação.

\section{Information quality perception}

\begin{abstract}
Attributes or concepts of information quality has been used by some authors to understand it better. Others criticize the proposal of attributing quality to information. In this paper, some provocative ideas concerning the perception of these attributes by the information user are discussed. There is also a list of attributes with their respective understandings, classified by the approach perspective, whether focused on the product or on the user. If focused on the product, the information is considered a good or thing; if focused on the user, it is appreciated according to the user's needs. In a research with a sample of information users who work in estate management, the author did not find a clear understanding of the concepts about information quality attributes. When some concepts or attributes were presented to these users, it was observed that their perception got closer to the common sense and was strongly influenced by the use of synonyms.
\end{abstract}

\section{Keywords}

Information science. Information quality. Information quality perception.

\section{INTRODUÇÃO}

Na prática profissional de diversos atores sociais, há sempre o contato com a informação. Ela é imprescindível em qualquer área do conhecimento, especialmente na acadêmica. Está, porém, tão incorporada ao nosso cotidiano, que não paramos para pensar sobre a sua qualidade. E até nos surpreendemos: a informação tem qualidade? Uns dizem que sim; outros, que não.

\section{ENTENDENDO O QUE É INFORMAÇÃO}

Sem delongas, cabe uma reflexão sobre o que é informação. Há alguns tantos entendimentos. Le Coadic (1996, p.5) aceita que a informação seja um conhecimento inscrito sob a forma escrita, oral ou audiovisual. Ela comporta um elemento de sentido e é transmitida a um ser consciente por meio de uma mensagem inscrita.

Bukland (1991, p. 351-360), por sua vez, identifica três usos principais da palavra informação: informação como processo, informação como conhecimento; informação como coisa. Como processo, a informação muda o conhecimento de alguém e é situacional. A ação de relatar ou o fato de começar a relatar sobre algo caracteriza a informação como processo, é o ato de informar um objeto, um documento, um dado, um fato, um evento. A relevância do dado ou fato é situacional e depende do nível de conhecimento de quem recebe a informação no momento da recepção. A informação como conhecimento tem uma de suas formas quando reduz as incertezas. O conhecimento comunicado refere-se a algum fato, assunto ou evento dado como notícia, informado, dito, que reflete no conhecimento, sendo, entretanto, intangível, não podendo ser tocado ou medido. A informação como coisa se refere aos objetos que são considerados como sendo informativos em suas características físicas, tais como o dado e os documentos expressos, descritos ou representados por alguma forma física como o sinal, o texto ou a comunicação desses.

Outros tantos entendimentos podem ser encontrados na literatura, mas o que tem prevalecido é que o dado propicia a informação que propicia o conhecimento. 


\section{QUALIDADE DA INFORMAÇÃO}

Autores e usuários perguntam-se por que é tão difícil a apreensão do conceito da qualidade da informação. Em primeiro lugar, a qualidade é um desses substantivos abstratos, um desses constructos de entendimento rápido por meio do senso comum, mas de complexo entendimento, quando se busca definição mais rigorosa, quando, na construção de uma teoria se procura relacionálo a outras variáveis. Segundo a definição do dicionário (FERREIRA, 1975, p.1165), "a qualidade é uma das categorias fundamentais do pensamento: maneira de ser que se afirma ou se nega de uma coisa”. Ora, as categorias fundamentais são exatamente as mais difíceis de serem definidas, sendo normalmente utilizadas para definir outras. É também definida como "aspecto sensível e que não pode ser medido das coisas”. Nesta última definição, reside um dos principais problemas do conceito qualidade da informação. Como avaliá-la? Com o acúmulo exponencial da quantidade de informações disponíveis no último século e com o desenvolvimento espetacular dos processos técnicos de registro e de acesso a essas informações, passamos a viver um problema que se tornou fundamental, qual seja, o de selecionar no imenso estoque de informações atualmente existente, aquelas que têm qualidade. Mas quais são os parâmetros a serem observados no processo de seleção para verificar se uma informação tem boa qualidade? Será que, como diz Marchand (1990), pode-se deixar o problema exclusivamente a cargo do usuário da informação, com a suposição de que qualidade da informação seja semelhante à beleza: "Eu a reconheço quando a vejo"?

Em contrapartida, ao descrever os parâmetros ou atributos relacionados ao conceito (na verdade dimensões usadas para definir a qualidade) e quantificá-los, está-se tentando contornar o problema esboçado na definição anteriormente citada. É claro que o conceito, o constructo, não se presta a medidas ou testes. A saída normalmente utilizada pela ciência é então, por meio, de definição operacional, levá-lo para o nível das observações quantitativas. Ocorre que essa operacionalização nem sempre é satisfatória, dependendo do estágio de conhecimento do conceito, da teoria na qual está envolvido e das possibilidades instrumentais de medidas. Além da dificuldade básica descrita anteriormente, a tarefa de desenvolvimento da noção de qualidade da informação no campo da ciência da informação parece configurar dificuldade mais profunda, como pode ser comprovado pelo trabalho de desconstrução desse conceito feito por Nehmy e Paim (1998) e por Nehmy (1996).
A partir do momento em que os conceitos vão completando seus ciclos de adaptação, a partir de construções teóricas em cada campo, institui-se a especificidade dos conceitos para aquele campo. Como ciência em fase de estruturação, a ciência da informação estaria então tentando readaptar, construir base teórica que inclua o conceito de qualidade, para aplicá-lo ao seu objeto principal, a informação. Parece não haver, portanto, no atual momento de desenvolvimento da ciência da informação, consenso definitivo para a qualidade da informação. Para Valls e Vergueiro (1998, p. 47), "especificamente na área da ciência da informação, nota-se a inexistência de uma base teórica sólida que permita um estudo aprofundado do tema". De fato, Nehmy (1996, p. 5-6) atesta que:

A análise permite concluir que, do ponto de vista epistemológico, a noção de qualidade da informação, tal como definida, é uma noção vaga, imprecisa, assumindo a característica de um conceito obstáculo. ... conclui-se definitivamente que a 'qualidade da informação' - ou noções correlatas analisadas - deva ser recusada enquanto conceito científico.

Nehmy e Paim (1998, p.36) dizem que:

Tomando como principal referencial as categorias analíticas de Gaston de Bachelard - estádios de um conceito e obstáculo epistemológico -, a leitura da noção 'qualidade da informação', tal como é abordada na literatura, revela que se trata de uma noção vaga, imprecisa, situando-se muito próxima ao entendimento do senso comum.

Em outro artigo, Paim, Nehmy e Guimarães (1996, p.112) afirmam que:

... não há consenso na literatura sobre definições teóricas e operacionais da qualidade da informação. Há uma alusão recorrente entre autores interessados no tema de que as definições de qualidade da informação são ambíguas, vagas ou subjetivas.

Mesmo assim, a ciência da informação faz tentativas de estudos da qualidade da informação em uma dicotomia que se revela na literatura em abordagens que tendem a avaliar os sistemas de informação (a qualidade sendo um desses critérios de avaliação) a partir do próprio sistema (avaliando seu projeto, conteúdo, atendimento ao usuário), mais propícia à operacionalização dos conceitos e mais voltada para aspectos objetivos, baseada no produto, e abordagens que tendem a avaliar os sistemas 
de informação a partir de considerações centradas na pessoa e enfocando aspectos mais subjetivos, com base no usuário.

\section{Abordagem da qualidade da informação baseada no produto}

A abordagem baseada no produto, sob a análise da qualidade da informação, enfatiza a informação como coisa. Nessa linha, atribuem-se à qualidade da informação algumas dimensões ou atributos, tais como abrangência, acessibilidade, atualidade, confiabilidade, objetividade, precisão e validade. Esses atributos, que conferem multidimensionalidade à qualidade da informação, foram pesquisados por diversos autores e discutidos no artigo de Paim, Nehmy e Guimarães (1996), que demonstram a estreita inter-relação entre os mesmos.

A abrangência e a objetividade constantes das obras consultadas são encontradas em trabalho de Casanova (1990), conforme Nehmy (1996, p. 30), que cita esses atributos como valores intrínsecos da informação.

$\mathrm{O}$ atributo acessibilidade foi mencionado por Buckland e Atkinson, citados por Valls e Vergueiro (1998, p.49). Para Buckland, citado por Valls e Vergueiro (1998, p. 49), o acesso é particularmente importante para a área da informação, basicamente preocupada com a prestação de serviços. Atkinson, em citação de Valls e Vergueiro, (1998, p. 49), vê que o propósito fundamental de todos os serviços de informação tem sido e sempre será reduzir o tempo necessário para que clientes-usuários específicos ganhem acesso àquela informação de que necessitam para atingir seus objetivos pessoais ou de trabalho institucional.

A atualidade "implica consonância com o ritmo de produção da informação, ou seja, opõe-se à obsolescência" (PAIM; NEHMY; GUIMARÃEE, 1996, p. 116).

A confiabilidade "significa credibilidade no conteúdo e na fonte de informação. Relaciona-se com a idéia de autoridade cognitiva - prestígio, respeito, reputação da fonte, autor ou instituição. A confiabilidade assemelhase a uma espécie de fé" (PAIM; NEHMY; GUIMARÃES; 1996, p. 116).

A precisão "tem o sentido aproximado da exatidão, correção, o que nos remete à forma de registro fiel ao fato representado (PAIM; NEHMY; GUIMARÃES; 1996, p. 116).
O conceito de validade "pressupõe integridade da fonte de informação e forma de registro fiel ao fato que representa" (PAIM; NEHMY; GUIMARÃES; 1996, p. 116).

O termo qualidade foi, a partir da década de 70, muito usado na administração e nos negócios, gerando o método do controle da qualidade total (CQT) ou gestão da qualidade total (GQT). Nessa linha, Campos (1992, p. 2) diz que:

um produto ou serviço de qualidade é aquele que atende perfeitamente, de forma confiável, de forma acessível, de forma segura e no tempo certo, às necessidades do cliente.

Nessa linha, para a Associação Brasileira de Normas Técnicas (ABNT, 1994, p. 3-4), citada por Valls e Vergueiro (1998, p.49), a qualidade tem a seguinte definição:

Totalidade de características de uma entidade que lhe confere a capacidade de satisfazer às necessidades explícitas e implícitas.... em algumas referências a qualidade é definida como 'adequação ao uso' ou 'adequação ao propósito' ou 'satisfação do cliente' ou 'conformidade aos requisitos'.

Entretanto, Paim e Nehmy (1998, p.87) alertam para o fato de que:

Apesar de as abordagens teóricas sobre o tema da qualidade ou valor da informação não se aterem a tais pressupostos - pelo menos de uma forma direta ou explícita, por não aderirem à proposta da qualidade total enquanto marco conceitual de referência -, a contaminação pelas idéias dominantes no ambiente da administração de empresas do qual originam as discussões da vertente da informação gerencial aparenta ser inevitável.

\section{Abordagem da qualidade da informação baseada no usuário}

Segundo Saracevic (1996, p.46), nos anos 70, o paradigma da recuperação da informação deslocou-se em direção à contextualização mais ampla, voltando-se para os usuários e suas interações, refletindo compreensão mais aprofundada do problema. Nessa década e na seguinte, vários autores começam a questionar a limitação da abordagem objetivista, da consideração dos sistemas de informação a partir do lado técnico, da eficácia da recuperação da informação. 
Em 1986, Dervin e Nilan (1986) fazem um recenseamento dessas questões e proposições que estavam sendo discutidas e que, embora apontassem para vários caminhos, de um modo geral passavam a tomar o usuário como centro das atenções nas considerações sobre os sistemas. Os autores apontam a tensão visível presente na literatura entre a pesquisa na ciência da informação e a prática. Em geral, os autores clamavam por pesquisas sobre necessidades e usos da informação, e a reorientação dos serviços para os usuários sob o argumento de que as pesquisas disponíveis não estavam provendo base para essa reorientação. Entre os temas para os quais se pedia maior atenção dos pesquisadores, estavam:

- a necessidade de melhor atendimento à clientela (usuários) baseada, entre outras coisas, na verificação da baixa utilização dos sistemas de informação;

- a centralidade das necessidades e usos da informação definidas a partir do usuário. A esse respeito citam Mick et alii (1980):

Uma transição efetiva para a era da informação vai requerer uma mudança de sistemas de informação direcionados para a tecnologia e o conteúdo, para sistemas de informação direcionados para o usuário.

De modo geral, os atributos da qualidade da informação que a literatura associa ao usuário são adequação da indexação e classificação (atributo do sistema), eficácia, eficiência da recuperação (atributo do sistema), impacto, relevância, utilidade, valor esperado, valor percebido e valor de uso.

A adequação da indexação e classificação como atributo do sistema de informação é associada à qualidade da informação quando considerada uma 'relevância objetiva', conforme Nehmy e Paim (1998, p. 42):

... a 'relevância objetiva' que trabalha a partir de hipóteses de adequação dos tópicos de indexação e classificação de documentos às questões da pesquisa bibliográfica, onde a questão da pesquisa é definida pela representação de uma necessidade de informação. Em tal perspectiva a relevância é a medida da adequação entre uma fonte e um destinatário.

Outro ponto prezado pela biblioteconomia e pela ciência da informação é a eficiência na recuperação da informação dentro de um sistema. A documentação e a recuperação não costumam operar de modo direto o termo de qualidade e usam a expressão relevância para refletir a noção principal em torno da avaliação da informação (NEHMY; PAIM, 1998, p. 42).

A eficácia da informação é lembrada por Saracevic, conforme citações registradas em Nehmy e Paim (1998, p. 41):

englobando a idéia de qualidade, no sentido de se atender às novas pressões que a sociedade da informação e o correlato desenvolvimento da tecnologia vêm fazendo à ciência da informação.

E em Paim, Nehmy e Guimarães (1996, p. 116):

Eficácia significa o grau de adequação da informação na solução do problema do sujeito-usuário da informação. A eficácia decorre do uso da informação, isto é, a informação é eficaz se contribui para algum resultado positivo para o sujeito da ação, como, por exemplo, tomada de decisão adequada.

O impacto da informação foi mencionado por Saracevic, conforme citação de Nehmy (1996, p. 42), como alusão, na maioria das vezes de forma oral, aos critérios para a abordagem da avaliação da informação:

... a análise de citações como critério de qualidade da produção científica acaba por se referir aos aspectos de utilidade ou relevância julgadas pelo usuário da literatura de determinada comunidade científica. Harter e Hooten (1992) tomam as palavras qualidade, utilidade ou relevância como termos equivalentes, ao relatarem resultados de pesquisa sobre a relação entre citações e financiamento em publicações da área da ciência da informação. Nesse caso, a medida de qualidade (ou de seus termos equivalentes) será dada pelo número de citações recebidas pelo artigo científico" (NEHMY, 1996, p. 44).

A utilidade é citada juntamente com relevância conforme anteriormente enfatizado. Entretanto, Cooney (1991, p. 185-186) afirma que:

O valor da informação deriva do valor de outro fenômeno, para cuja manutenção e desenvolvimento a informação contribui. .... A informação também pode ser analisada da perspectiva de quem ou para que ela será útil. ... A utilidade da informação para as empresas reside essencialmente na sua contribuição para decisões lucrativas. 
Nessa linha de pensamento, Nehmy e Paim (1998, p. 40) citam A. J. Repo em artigo no qual o autor acentua que, em geral, os estudos vindos da ciência da informação tratam o valor de uso (caráter cognitivo). O valor de uso, que leva em consideração o usuário, o uso e os efeitos no trato da informação, daria uma abordagem com ênfase no usuário. Esse valor de uso seria subdividido em valor esperado e valor percebido, não definidos pelos autores, e que só seriam apreendidos por medidas qualitativas.

\section{PERCEPÇÃO DA QUALIDADE DA INFORMAÇÃO}

Em março de 2001, trabalhando com um grupo de discussão* (ou grupo focal) com vistas a subsidiar a sua tese de doutorado, Oleto (2003) teve oportunidade de caracterizar e entender a percepção do usuário quanto aos conceitos da qualidade da informação. Estes muito vinculados à prática cotidiana do trabalho, nada a partir de elucubrações mentais. Mas ficou evidente que os conceitos de qualidade da informação são tratados de forma pragmática e combinados entre si, não havendo a clareza para a distinção individual e própria de cada um dos partícipes do grupo. A realidade prática é que leva à construção do conceito. E é relevante registrar que o grupo de discussão deixou claro que os conceitos de qualidade da informação não são percebidos de forma individual; não se separa ou isola cada um dos conceitos no raciocínio dos usuários. Todos esses atributos da qualidade da informação se misturam, e, para os usuários, é tênue a distinção inequívoca entre eles. Foi possível distinguir um a um, desde que se usasse exemplo empírico que surgisse e esclarecesse a distinção entre os conceitos. Os usuários, segundo o grupo de discussão, não foram capazes de relatar de forma distinta, separada, sem superposição, os conceitos teóricos da qualidade da informação. A prática e o modo de uso da informação dão ao usuário o entendimento do conceito acadêmico, que ele intuitivamente trabalha sem conhecer a teoria que constrói as definições e os conceitos.

Assim, a qualificação da informação com alguns atributos não é evidente para o usuário, e ele parece não escolher sua informação pelos conceitos. Trabalha-os de forma intuitiva, usando do senso comum e da sinonímia para manifestar sua percepção da qualidade da informação. Não há evidências de familiaridade ou de conhecimento explícito com o tema. Quando muito, um conhecimento tácito, desorganizado e pautado por comparações e sinônimos.

\footnotetext{
* O grupo foi formado por nove usuários da informação que trabalhavam no mercado imobiliário em Belo Horizonte - MG.
}

\section{CONCLUSÃO}

A percepção da qualidade não é nítida por parte do usuário da informação. Fica mais aproximada do conhecimento popular em vez do conhecimento científico. Talvez seja pela própria falta de conceitos claros que sustentem interpretações inequívocas da qualidade da informação (se isto for possível). Mas tal busca, a da perfeita e precisa definição dos atributos que qualificam a informação, permitindo a inquestionável percepção do usuário, ainda não aponta para paradigmas a que se possa recorrer com segurança (ainda se isto for possível). Os usuários pesquisados sentiram a necessidade de serem estimulados com conceitos que os aproximassem do referencial teórico e que, também, pudessem ser traduzidos para o cotidiano profissional. A experiência de pensar a informação a partir da sua qualidade ainda não havia sido vivida por estes usuários. Essa experiência deixou caladas as indagações: é possível selecionar ou trabalhar uma informação escolhendo-a a partir dos atributos/ conceitos da qualidade da informação? É possível definir quantos e quais atributos/conceitos são suficientes para garantir qualidade a uma informação?

Artigo submetido em 24/10/2005 e aceito em 10/07/2006.

\section{REFERÊNCIAS}

ASSOCIAÇÃO BRASILEIRA DE NORMAS TÉCNICAS - ABNT. ABNT/CB-25 - gestão da qualidade e garantia de qualidade - terminologia NBR ISO 8402. Rio de Janeiro, 1994, 15 p.

BUCKLAND, Michael K. Information as thing. Journal of the American Society for Information Science, v. 42, n. 5, p. 351-360, June 1991.

CAMPOS, Vicente Falconi. TQC: controle de qualidade total (no estilo japonês). Belo Horizonte: Fundação Christiano Ottoni: UFMG, 1992a.

CASANOVA, M. B. Information: the major element for change. In: WORMELL, I. (Ed.). Information quality: definitions and dimentions. London: Taylor Graham, 1990. p.42-53.

COONEY, James P. Qual o real valor da informação?. Revista Escola de Biblioteconomia/UFMG, Belo Horizonte, v. 20, n. 2, p. 176-190, jul./ dez. 1991.

DAVENPORT, Thomas H. Ecologia da Informação: por que só a tecnologia não basta para o sucesso na era da informação. 6. ed. São Paulo: Futura, 1998.

DERVIN, Brenda, NILAN, Michael. Information needs and uses. Annual Review of Information Science and Technology, v. 21, p. 3-32, 1986.

FERREIRA, Aurélio Buarque de Holanda. Novo dicionário da língua portuguesa. Rio de Janeiro: Nova Fronteira, 1975.

LE COADIC, Yves-François. A ciência da informação. Brasília: Briquet de Lemos Livros, 1996.

MARCHAND, Donald. Managing Information quality. In: WORMELL,I. (Ed.). Information quality: definitions and dimensions. London: Taylor Graham, 1990, p.7-17. 


\section{Ronaldo Ronan Oleto}

MCGEE, James; PRUSAK, Laurence. Gerenciamento estratégico da informação: aumente a competitividade e a eficiência de sua empresa utilizando a informação como uma ferramenta estratégica. 12. ed. Rio de Janeiro: Campos, 1994.

MICK, C. K. et al. Toward usable user studies. Journal of the American Society for Information Science, v. 31, n. 5, p. 347-356, Sept. 1980.

NEHMY, Rosa Maria Quadro. Leitura epistemológico-social da qualidade da informação. 1996. Dissertação (Mestrado em Ciência da Informação)- Programa de Pós-graduação em Ciência da Informação, UFMG, Belo Horizonte, 1996.

; PAIM, Isis. A desconstrução do conceito de "qualidade da informação". Ciência da Informação, Brasília, v. 27, n. 1, p. 36-45, jan./abr. 1998.

OLETO, Ronaldo Ronan. A qualidade da informação na percepção do usuário em diferentes contextos informacionais. 2003. Tese (Doutorado)Universidade Federal de Minas Gerais, Escola de Ciência da Informação, Belo Horizonte, 2003.
PAIM, Isis, NEHMY, Rosa Maria Quadro. Questões sobre a avaliação da informação: uma abordagem inspirada em Giddens. Perspectivas em Ciência da Informação, Belo Horizonte, v. 3, n. 1, p. 81-95, jul./dez. 1998.

; GUIMARÃES, César Geraldo. Problematização do conceito "qualidade" da informação. Perspectivas em Ciência da Informação, Belo Horizonte, v. 1, n. 1, p. 111-119, jan./jun. 1996.

SARACEVIC, T. Ciência da informação: origem, evolução e relações. Perspectivas em Ciência da Informação, Belo Horizonte, v. 1, n. 1, p. 41 62, jan./jun. 1996.

VALLS, Valéria Martin, VERGUEIRO, Waldomiro C. S. A gestão da qualidade em serviços de informação no Brasil: uma revisão da literatura. Perspectivas em Ciência da Informação, Belo Horizonte, v. 3, n. 1, p. 47-59, jan./jun. 1998. 